\title{
El valor del Registro Nacional de Procedimientos del CACl
}

\section{The value of the National Registry of Procedures of the CACl}

Revista Argentina de Cardioangiología Intervencionista 2018;9(2):109. Doi: 10.30567/RACI/201802/0109-0109

El Registro voluntario Nacional de Procedimientos, que se realiza anualmente desde el año 2007, tiene una importancia fundamental para el manejo general de nuestra especialidad.

Determinar fehacientemente el número de procedimientos diagnósticos y terapéuticos tanto en territorio coronario como no coronario, en pacientes pediátricos y en adultos, a lo largo y a lo ancho de nuestro país, nos permite tener el mapa de nuestro contexto, en el tratamiento de las patologías cardiovasculares en general.

Con esta información resultará más sencillo sentarnos con los financiadores de la salud tanto pública como privada a discutir qué parte de la gran masa de atención pasa por nuestras salas. Permitiéndonos plantear cuánto hay que destinar en salud para realizar las prácticas de nuestra especialidad, basándonos en números reales.

Sin embargo, a pesar de lo relevante que resulta contar con esta información completa, resulta llamativa la escasa adherencia al Registro.

A pesar del trabajo continuo de la Oficina de Registros y de su coordinador, el Dr Omar Santaera, los centros que aportan sus datos al Registro, solo representan el 30\% en los últimos años.

Los datos correspondientes al año 2016, presentados durante las Jornadas Científicas y Gremiales CACI 2017 llevadas a cabo en la ciudad de Salta, muestran que de 335 centros, solo 96 aportaron los datos correspondientes a su actividad. Esto representa el $28,7 \%$ de los servicios de Hemodinamia del país.

Este bajo porcentaje de centros participantes contrasta de manera notable, por ejemplo, con el Registro de la Sección de Hemodinámica y Cardiología Intervencionista de la Sociedad Española de Cardiología, el cuál desde 1990 comunica anualmente la actividad intervencionista en España. Al igual que nuestro Registro Nacional de Procedimientos, el mismo es voluntario, no auditado y realizado a través de una base de datos on line. El contraste entre ambos registros se expresa en que mientras que al nuestro vuelcan sus datos en promedio el 30\% de los servicios de Hemodinamia del país, en el caso de España, el registro refleja la actividad anual del 100\% de los hospitales públicos y del $92 \%$ de los centros privados ${ }^{1}$, constituyendo cada uno de sus reportes la fuente más actual, completa y rigurosa de la actividad intervencionista del país.

Desde esta Comisión Directiva, estamos firmemente dispuestos a revertir esta situación. Hemos sumado a la Oficina de Registros, a los doctores Leandro Lasave, Gerardo Nau y Gustavo Pedernera, quienes manifestaron su interés y vocación de trabajar en la misma, para que junto a los doctores Santaera y Torresani instrumenten los medios para revertir en este año la escasa participación y compromiso, y hacernos sentir a todos partícipes necesarios y beneficiarios de disponer de esta información indispensable para conocer la realidad de la Cardioangiología Intervencionista en nuestro país.

No quiero dejar pasar la oportunidad de mencionarles que, a iniciativa del Área de Investigación del Colegio, durante este año se iniciará el Registro Argentino de Angioplastia Coronaria - RAdAC 2, coordinado en forma conjunta por el CACI, el Consejo de Hemodinamia de la SAC y el Comité de Hemodinamia de la FAC.

Recordemos que entre Mayo del 2010 y Febrero del 2012 el estudio RAdAC, del que participaron 67 centros con Servicio de Hemodinamia y en el que se incluyeron 3102 pacientes, dio lugar a la presentación y publicación de diversos trabajos que surgieron del mismo.

El objetivo fundamental de RAdAC 2, está dirigido a conocer como estamos realizando nuestras intervenciones coronarias en la actualidad, ya que es notable en los últimos años la evolución de las indicaciones y recursos disponibles. Los invito a todos a que, como miembros del Colegio, nos comprometamos a tener una participación activa en estos y otros registros relacionados con nuestra especialidad, los que de esa manera representarán el abanico completo de la realidad de la práctica de la Cardioangiología Intervencionista en nuestro país.

Dr. Aníbal Damonte Presidente del CACI

\section{BIBLIOGRAFÍA}

1. Registro Español de Hemodinámica y Cardiología Intervencionista. XV informe oficial de la Sección de Hemodinámica y Cardiología Intervencionista de la Sociedad Española de Cardiología. Rev EspCardiol 2006;59(11):1146-64. 